\title{
Searches for Higgs Bosons at the Tevatron
}

\author{
Anton Anastassov \\ Rutgers University, NJ 08854, USA \\ On behalf of the CDF and DØ Collaborations
}

\begin{abstract}
We present the results of searches by the CDF and DØ Collaborations for Higgs boson production in $p \bar{p}$ collisions at $\sqrt{s}=1.96 \mathrm{TeV}$. The searches are performed in a range of production and decay channels predicted by the Standard Model and its minimal supersymmetric extension (MSSM) with data samples corresponding to $200-950 \mathrm{pb}^{-1}$. In the absence of signal, the results are used to set upper limits on Higgs production cross sections times branching fractions.
\end{abstract}

Keywords: Higgs, Standard Model, SUSY, MSSM, CDF, DØ

PACS: $12.60 . \mathrm{Fr}, 12.60 . \mathrm{Jv}, 14.80 . \mathrm{Bn}, 14.80 . \mathrm{Cp}$

\section{INTRODUCTION}

In the past decades high energy physics has made a tremendous progress in building a consistent picture of the properties of the fundamental particles and their interactions. Despite the successful developments on both the theoretical and experimental fronts, there are still many areas that provide exciting research opportunities. One of the outstanding questions is the dynamics of electroweak (EW) symmetry breaking and the origin of particle masses. In the Standard Model (SM), EW symmetry is spontaneously broken through the introduction of a doublet of self-interacting complex scalar fields with non-zero vacuum expectation values (Higgs mechanism). A consequence of this scenario is the existence of a new scalar boson (Higgs boson). The interactions of the Higgs particle with fermions and bosons are well defined as a function of its mass $\left(m_{H}\right)$, however, the mass itself is not predicted by the theory. Experimental bounds on the mass are set by direct Higgs searches at LEP [1], and global fits of electroweak measurements [2]. The combined results of the two approaches constrain $m_{H}$ at $95 \%$ confidence level to the range $114.4-199 \mathrm{GeV}$.

Many extensions of the SM, including supersymmetric (SUSY) theories, require more than one Higgs field doublets, leading to extended Higgs sectors containing both neutral and charged bosons. They provide additional possibilities for experimental searches.

The CDF and DØ collaborations have comprehensive and focussed research programs that include searches for evidence for Higgs bosons as predicted in the SM and extended theories. The results presented in this paper are based on data collected with the CDF and $\mathrm{D} \emptyset$ detectors in Run 2 of the Fermilab Tevatron. The Tevatron is a proton antiproton $(p \bar{p})$ collider running at a center-of-mass energy $\sqrt{s}=1.96 \mathrm{TeV}$. CDF and D $\varnothing$ are multi-purpose detectors build around the two $p \bar{p}$ interaction points at the Tevatron ring. Detailed description of the detectors and their performance can be found in Ref. [3] and Ref. [4]. 


\section{SEARCHES FOR SM HIGGS BOSONS}

This group of results includes searches for Higgs bosons $(H)$ with properties as predicted by the SM. However, the results are also relevant for certain SUSY scenarios that include a SM-like Higgs as part of the particle spectrum. The main production mechanisms of $H$ at the Tevatron are gluon fusion, and production in association with a $W$ or $Z$ boson. The branching fractions of Higgs decays depend strongly on $m_{H}$. The search strategies are driven by the most promising combinations of Higgs production and decay modes in each mass region (Figure 1), and take into account the expected background levels.
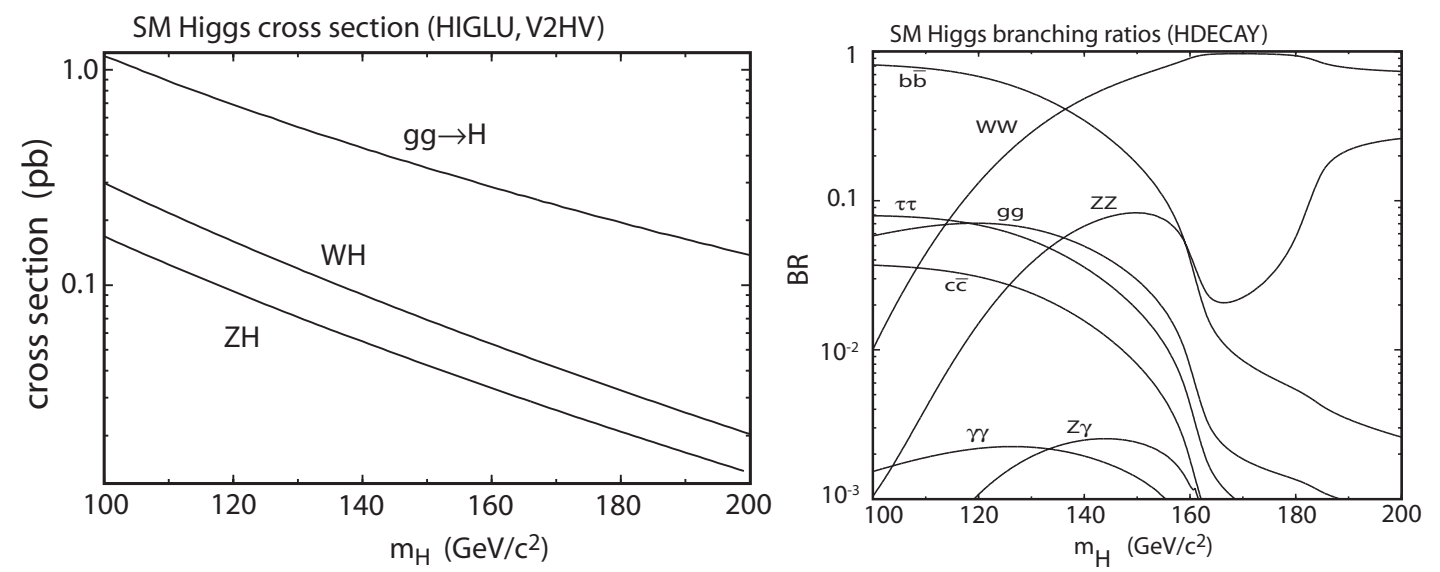

FIGURE 1. Dominant production cross sections at the Tevatron [5] and branching fractions [6] of the SM Higgs boson as a function of $m_{H}$ [7].

The decay $H \rightarrow b \bar{b}$ is dominant for $m_{H}<135 \mathrm{GeV}$ ("low-mass" region). Identification of the jets produced by the $b$-quarks is achieved by applying $b$-tagging algorithms. Btagging at $\mathrm{CDF}$ and $\mathrm{D} \varnothing$ takes advantage of the long life of $b$-quarks, and relies either on the reconstruction of a displaced secondary vertex, or on the probability that the tracks associated with the jet do not originate from the point of primary interaction. For higher masses Higgs bosons decay predominantly to $W W^{*}$, where one of the $W$ 's can be offshell.

Due to the large number of explored channels we give only brief descriptions of the SM searches at the Tevatron and present the results in a single combined plot at the end of this Section. Details can be found in the provided references and in the dedicated CDF and $\mathrm{D} \varnothing$ contributions to these Proceedings.

$\underline{W H \rightarrow l v b \bar{b}}$

This search channel is one of the most effective tools for probing the low-mass Higgs region. The detector signature of signal events is one electron or muon with large transverse momentum $\left(p_{T}\right)$, two jets, and large missing transverse energy ${ }^{1}\left(\mathscr{E}_{T}\right)$

\footnotetext{
${ }^{1}$ Missing transverse energy is calculated from the imbalance of energy deposition in the calorimeter
} 
carried by the neutrino. The CDF and D $\varnothing$ collaborations have both performed searches in this channel using similar event selection criteria. Events of interest must contain an electron or a muon with $p_{T}>20 \mathrm{GeV}$, and at least one of the jets must be $b$-tagged. CDF requires that the transverse energy of each of the two jets is above $15 \mathrm{GeV}$, and the missing transverse energy in the event $\left(\mathbb{E}_{T}\right)$ to be greater than $20 \mathrm{GeV}$. The corresponding selections at $\mathrm{D} \emptyset$ are jet $E_{T}>20 \mathrm{GeV}$, and $\mathbb{E}_{T}>25 \mathrm{GeV}$. After topological cuts the dominant backgrounds in the selected event samples are from $W b \bar{b}, W c \bar{c}$, and $t \bar{t}$ production. Both searches take advantage of the difference in the signal to background ratio in the single- and double-tagged event samples and treat them as separate channels for added sensitivity. In the $\mathrm{D} \varnothing$ analysis, the samples are also divided based on the flavor of the lepton in the event.

Signal events are looked for in the invariant di-jet mass distributions. No evidence for $W H$ production was found in the analyzed data samples of $695 \mathrm{pb}^{-1}$ (CDF), and $378 \mathrm{pb}^{-1}(\mathrm{D} \varnothing)$, and the results of the searches are used to set $95 \%$ CL upper limits on the product of production cross section times branching fraction $\sigma(p \bar{p} \rightarrow W H) \times B R(H \rightarrow$ $b \bar{b})$ as a function of Higgs mass. For $m_{H}=115 \mathrm{GeV}$ the limits set by CDF (DØ) are $3.6 \mathrm{pb}$ $(2.5 \mathrm{pb})$.

\section{$\underline{Z H \rightarrow v \bar{v} b \bar{b}}$}

The detector signature of signal events in this search mode is two jets from the Higgs decay, and missing transverse energy due to the neutrinos produced in $Z \rightarrow v \bar{v}$. The backgrounds are from di-boson production, $W / Z+$ jets, $t \bar{t}$, and multi-jet events. For background suppression CDF and D $\varnothing$ utilize vetoes on extra jets and leptons in the event, and requirements on the relative directions of the jets and $\mathbb{E}_{T}$. The event selection at DØ requires $\mathbb{E}_{T}>50 \mathrm{GeV}$, two jets with $E_{T}>20 \mathrm{GeV}$, and sum of the jets transverse energy less than $240 \mathrm{GeV}$. The data is divided into single and double-tagged event subsamples for added sensitivity. The search at CDF requires $\mathbb{E}_{T}>70 \mathrm{GeV}$, and two jets with $E_{T}>60,25 \mathrm{GeV}$. Signal evidence is sought by looking for excess of events in regions of the di-jet mass distributions over the background expectation. The boundaries of the selected mass regions are varied depending on the probed Higgs mass. Using a data sample of $289 \mathrm{pb}^{-1} \mathrm{CDF}$ set a limit $\sigma(p \bar{p} \rightarrow Z H) \times B R(H \rightarrow b \bar{b})<4.8 \mathrm{pb}$ at 95\% CL for $m_{H}=115 \mathrm{GeV}$. The corresponding result from $\mathrm{D} \varnothing$ with $260 \mathrm{pb}^{-1}$ [8] is $3.2 \mathrm{pb}$.

One of the interesting features of the $b \bar{b}+\mathbb{E}_{T}$ detector signature is that its sensitivity goes beyond the $Z H \rightarrow v \bar{v} b \bar{b}$ channel. Due to limited detector coverage and particle identification efficiency, a substantial fraction of the $W H \rightarrow l v b \bar{b}$ events will exhibit the same signature. $\mathrm{D} \varnothing$ has taken advantage of this feature and sets a 95\% CL upper limit on $\sigma(p \bar{p} \rightarrow W H) \times B R(H \rightarrow b \bar{b})$. The result for $m_{H}=115 \mathrm{GeV}$ is $7.5 \mathrm{pb}$.

\section{$\underline{t \bar{t}} H \rightarrow W W b \bar{b} b \bar{b}$}

The searches for Higgs production in association of a $t \bar{t}$ pair requires examination of a complex final state. The decay chain $t \bar{t} H \rightarrow W W b \bar{b} b \bar{b} \rightarrow l v q \bar{q} b \bar{b} b \bar{b}(l=e, \mu)$ is seen as the best compromise between signal efficiency and background suppression. The search for $t \bar{t} H$ production at CDF selects events with one lepton with $p_{T}>20 \mathrm{GeV}$, at least five jets with $E_{T}>15 \mathrm{GeV}$ (three or more jets must be $b$-tagged), and $\mathbb{E}_{T}>10 \mathrm{GeV}$. The dominant backgrounds are from $t \bar{t} b \bar{b}$ and $t \bar{t} c \bar{c}$ events. Only one event in a data 
sample of $320 \mathrm{pb}^{-1}$ satisfied the selection criteria, consistent with the expectation of $0.89 \pm 0.12$ background events. The upper limit on $\sigma(t \bar{t} H) \times B R(H \rightarrow b \bar{b})$ at $95 \%$ CL for $m_{H}=115 \mathrm{GeV}$ is $660 \mathrm{fb}$, about two orders of magnitude higher than the SM expectation. Nevertheless, this analysis provides valuable information for future searches at the LHC, where this channel is very promising.

$$
\underline{H \rightarrow W W^{*} \rightarrow l^{-} \bar{v} l^{+} v}
$$

With production cross section of $\sim 1.3-0.2 \mathrm{pb}$ for $m_{H}=110-200 \mathrm{GeV}$, gluon fusion is the dominant production mechanism of SM Higgs at the Tevatron. Due to the overwhelming $b \bar{b}$ production $g g \rightarrow H \rightarrow b \bar{b}$ is not a viable search mode. However, $g g \rightarrow H \rightarrow W W^{*} \rightarrow l^{-} \bar{v} l^{+} v(l=e, \mu)$ is very powerful for probing Higgs masses above $135 \mathrm{GeV}$. A detector signature of two isolated opposite-sign leptons and missing transverse energy efficiently discriminates against multi-jet events that are copiously produced at hadron colliders. CDF selects events with two leptons with $p_{T}>20,10 \mathrm{GeV}$, and $\mathbb{E}_{T}>m_{H} / 4$, where $m_{H}$ is the mass of the searched for Higgs boson. D $\emptyset$ requires leptons with $p_{T}>15,10 \mathrm{GeV}$, and $\mathbb{E}_{T}>20 \mathrm{GeV}$. Additional selection criteria provide further suppression of SM backgrounds, leaving $W W$ production as the dominant background in the searches. Due to spin correlations, the charged leptons produced in $H \rightarrow W W^{*} \rightarrow l^{-} \bar{v} l^{+} v$ tend to have smaller azimuthal separation angle $\Delta \phi$ than the ones from the $W W$ backgrounds, and CDF and D $\emptyset$ use $\Delta \phi$ as a signal/background discriminating variable.

The searches for $g g \rightarrow H \rightarrow W W^{*}$ at CDF with a data sample of $360 \mathrm{pb}^{-1}$ [9], and at DØ with a sample of $950 \mathrm{pb}^{-1}$ did not find signal evidence and set upper limits on the production cross section times branching fraction as a function of $m_{H}$. At $m_{H}=160 \mathrm{GeV}$ the obtained limits are $2.2 \mathrm{pb}(\mathrm{D} \emptyset)$, and $3.2 \mathrm{pb}(\mathrm{CDF})$.

\section{Summary of SM Higgs results at the Tevatron}

The size of the current data samples, and the sensitivity of the individual channels are not sufficient to exclude SM Higgs at any mass point. CDF and DØ have to rely on combining the results from all search modes to extend their reach. Figure 2 is a snapshot of how close (or far) we are from the SM predictions. The results are presented as a ratio of the exclusion limits obtained to date, to the predictions of the SM. The limits after combining all channels are also shown (separately for CDF and DØ). Although additional sensitivity will be gained after combining the CDF and D $\varnothing$ results, the plot shows that we need to implement many improvements to get closer to the theoretical predictions. Both Tevatron experiments are working on fuller utilization of multivariate methods for particle identification and signal/background separation. Improvements in the di-jet mass resolution will boost the sensitivity of $H \rightarrow b \bar{b}$ searches, and algorithms for more precise jet energy measurements are being developed by CDF and DØ. 


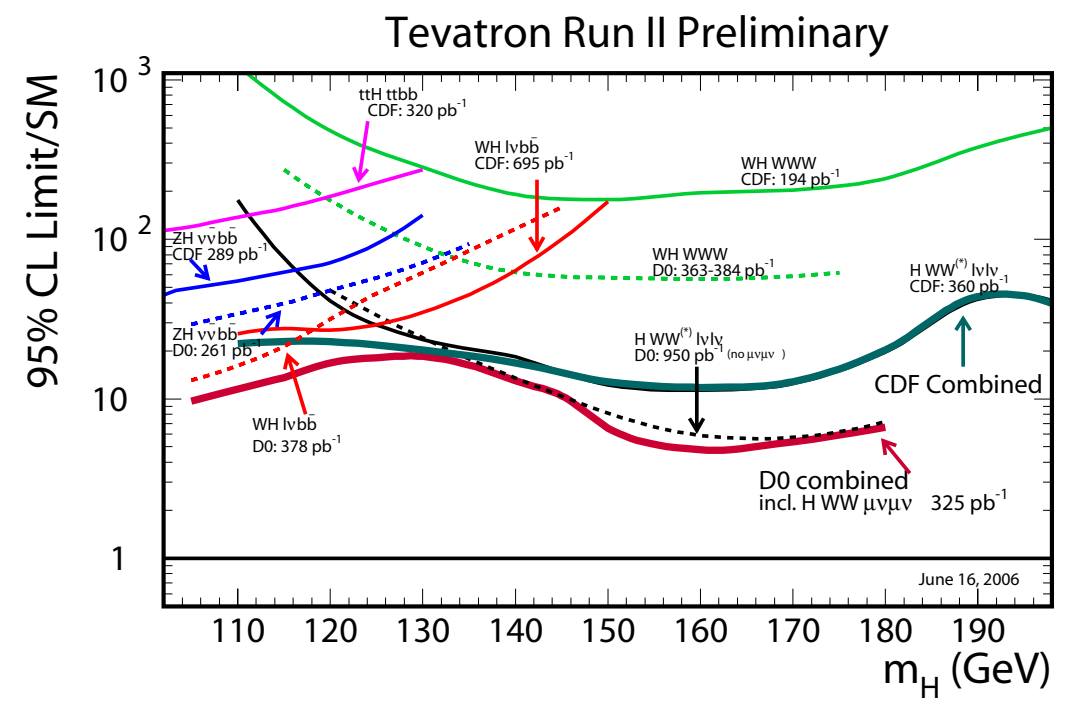

FIGURE 2. Ratio of the observed upper limits on Higgs production to the predictions of the SM.

\section{SEARCHES FOR HIGGS BEYOND THE STANDARD MODEL}

The Higgs sector in the Minimal Supersymmetric Extension of the Standard Model (MSSM) consists of two charged $\left(H^{ \pm}\right)$, and three neutral scalar bosons $(A, H, h)$. Assuming $C P$-invariance, one of the neutral bosons $(A)$ is $C P$-odd, and the other two are $C P$-even. At tree level the MSSM Higgs bosons are described by the mass of $A\left(m_{A}\right)$ and $\tan \beta=v_{u} / v_{d}$, where $v_{u}\left(v_{d}\right)$ is the vacuum expectation value of the components of the Higgs fields that couple to up-type (down-type) fermions. For $\tan \beta>30$, one of the $C P$ even bosons is nearly mass degenerate with $A$ and has similar Yukawa couplings, while the other is SM-like. The dominant production mechanism of neutral MSSM Higgs bosons at the Tevatron are $g g \rightarrow \phi$ (through a $b$-quark loop), and $b \bar{b} \rightarrow \phi(\phi=A, h / H)$. In the latter case, $\phi$ can be accompanied by $b$-quarks with large transverse momentum The enhanced couplings of $\phi$ to $b$-quarks lead to sizable production cross sections at large $\tan \beta$. The $\phi$ decays mostly to $b \bar{b}$ and $\tau^{+} \tau^{-}$with branching fractions $B R(\phi \rightarrow b \bar{b}) \sim 90 \%$ and $B R\left(\phi \rightarrow \tau^{+} \tau^{-}\right) \sim 9 \%$.

\section{Search for neutral MSSM Higgs bosons decaying to $b \bar{b}$}

The DØ collaboration has performed a search for $\phi \rightarrow b \bar{b}$ produced in association with one or two $b$-quarks with a data sample corresponding to $260 \mathrm{pb}^{-1}$ [10]. The analysis requires the presence of more than three, but less than five jets with $E_{T}>15 \mathrm{GeV}$ originating from the same vertex. At least three of the jets must be $b$-tagged. The search for signal evidence is performed by looking for enhancement in the invariant mass spectrum of the two leading jets. The dominant SM background in the search is from multi-jet production. The di-jet mass shape is obtained from multi-jet events with two $b$-tags, weighted by the $b$-jet misidentification probability. The remaining backgrounds are estimated from simulated events. This search found no evidence for neutral Higgs production, and sets 95\% CL upper limit on $\sigma(p \bar{p} \rightarrow \phi b(\bar{b}))$ - Figure 3. 

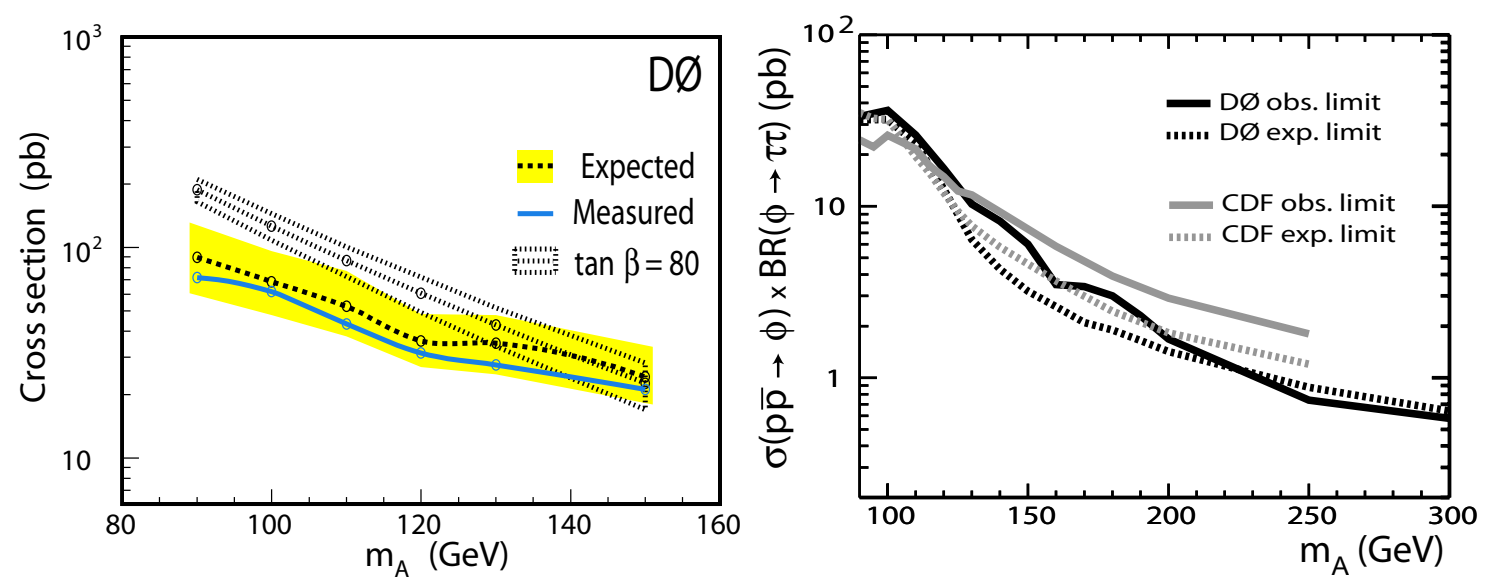

FIGURE 3. Limits on neutral MSSM Higgs production. The results from the D $\varnothing$ search for $\phi b(\bar{b}) \rightarrow$ $b \bar{b} b(\bar{b})$ with $260 \mathrm{pb}^{-1}$ are shown on the left plot. The results of the inclusive searches in the $\phi \rightarrow \tau^{+} \tau^{-}$ channel from $\mathrm{CDF}\left(310 \mathrm{pb}^{-1}\right)$ and $\mathrm{D} \emptyset\left(325 \mathrm{pb}^{-1}\right)$ are shown on the right.

Searches for neutral MSSM Higgs bosons decaying to $\tau^{+} \tau^{-}$

$\mathrm{CDF}$ and $\mathrm{D} \emptyset$ have developed efficient algorithms for detection of hadronic tau decays that allowed them to perform searches for inclusive neutral MSSM Higgs production and subsequent decay to tau pairs. The analyzed data samples correspond to integrated luminosity of $310 \mathrm{pb}^{-1}$ (CDF) and $325 \mathrm{pb}^{-1}$ (DØ). CDF has explored [11] final states with one tau decaying leptonically, and the other hadronically. In addition to these modes, DØ uses two leptonic decays through the detection of an electron, a muon, and $\mathbb{E}_{T}$ [12]. The dominant background in both searches is from $Z \rightarrow \tau^{+} \tau^{-}$events. The selected samples also contain events from $Z / \gamma^{*} \rightarrow e e, \mu \mu, W+$ jets, multi-jet, and diboson production. Limits on $\sigma(p \bar{p} \rightarrow \phi) \times B R\left(\phi \rightarrow \tau^{+} \tau^{-}\right)$are extracted from fits of the partially reconstructed di- $\tau$ mass (formed using the four-vectors of the visible tau decay products and $\mathbb{E}_{T}$ ). The results are presented as a fuction of $m_{A}$ in Figure 3.

The searches for neutral MSSM Higgs bosons in the $b \bar{b}$ and $\tau^{+} \tau^{-}$channels are complementary, as they exhibit different levels of sensitivity in different regions of the MSSM parameter space [13]. The obtained cross section upper limits can be interpreted as exclusions of parameter regions in the $\tan \beta$ vs $m_{A}$ plane. Four benchmarks are considered: the no-mixing and $m_{h}^{\max }$ scenarios [14], with higgsino mass parameter $\mu=$ $+200 \mathrm{GeV}$ and $\mu=-200 \mathrm{GeV}$. The corresponding upper limits on $\tan \beta$ as a function of $m_{A}$ are shown in Figure 4.

Search for charged MSSM Higgs Bosons in top-quark decays

Relatively light charged MSSM Higgs bosons $\left(m_{H^{ \pm}}<m_{t}-m_{b}\right)$ can be produced in top-quark decays via $t \rightarrow H^{+} b$. Possible contribution from this channel to top-quark 

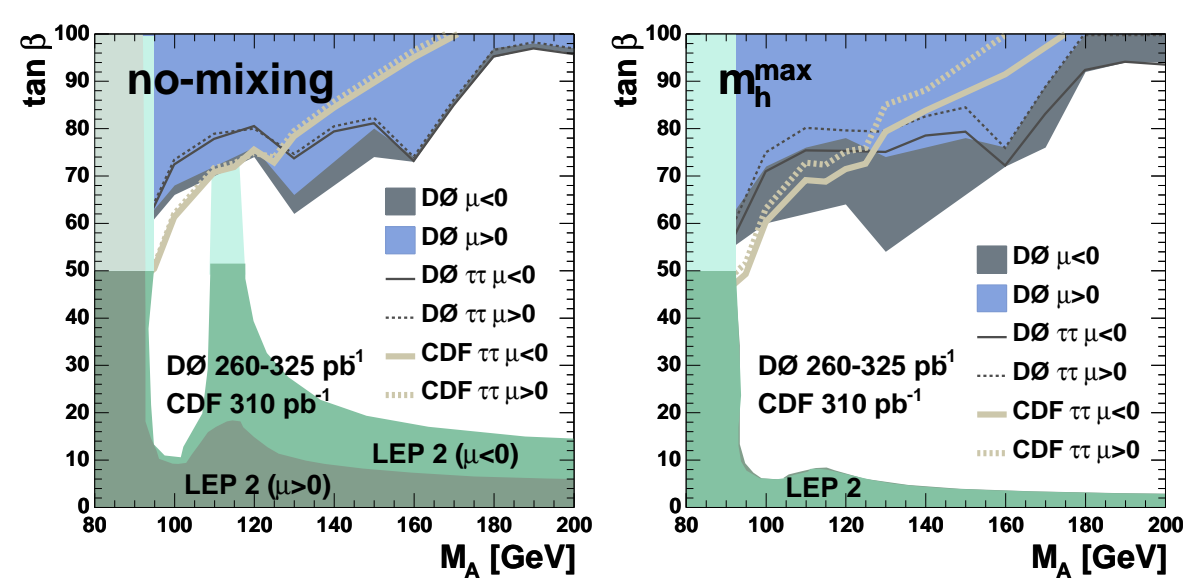

FIGURE 4. Excluded parameter space for the $m_{h}^{\max }$ and no-mixing benchmark scenarios from the searaches for neutral MSSM bosons.. The lines show the exclusions from the D $\varnothing$ and CDF searches for $\phi \rightarrow \tau \tau$. The excluded regions from combining the DO results in the $\phi \rightarrow \tau \tau$ and the $\phi b \bar{b} \rightarrow b \bar{b} b(\bar{b})$ channels are shown as shaded areas.

decays will compete with the SM process $t \rightarrow W^{+} b$. The final states produced in $H^{ \pm}$ decays depend on the MSSM parameters, and include $\tau^{+} v, c \bar{s}, t^{*} \bar{b} b$ and $W^{+} h$. Evidence for the existence of $H^{ \pm}$bosons will then appear as a deviation in the balance of observed topological final states in $t \bar{t}$ events from the SM expectation. The CDF analysis with $193 \mathrm{pb}^{-1}$ of data considers four topologies: $2 l+$ jets, $l+$ jets $(1 b$-tag), $l+$ jets $(\geq 2 b$-tags), $\tau$ $+l+$ jets $(l=e, \mu)$. No significant deviation from the SM expectation was observed. The result of the search were used to set limits on $\tan \beta$ as a function of $m_{H^{ \pm}}$- Figure 5. In the large $\tan \beta$ region, $H^{ \pm}$is expected to decay almost exclusively to a tau and a neutrino. In this scenario $B R\left(t \rightarrow H^{+} b\right)>0.4$ is excluded at $95 \% \mathrm{CL}$ for $80<m_{H^{ \pm}}<160 \mathrm{GeV}$.

Searches for doubly-charged Higgs in extended models

Doubly charged Higgs bosons are predicted by a range of models, including Little Higgs and Left-Right symmetric models. At the Tevatron, doubly-charged Higgs is expected to be pair-produced in the process $p \bar{p} \rightarrow Z / \gamma^{*} \rightarrow H^{++} H^{--}$. The $H^{ \pm \pm}$ decays to leptons are virtually unrestricted, and may involve lepton flavor violation. $\mathrm{CDF}$ has previously looked for doubly-charged Higgs in the decay modes $\mathrm{H}^{++} \rightarrow$ $e^{+} e^{+}, \mu^{+} \mu^{+}, e^{+} \mu^{+}$(here and below charge conjugate states are implied) and found no signal evidence. Using a data sample of $350 \mathrm{pb}^{-1}$, the range of investigated final states is extended to include $e^{+} \tau^{+}$and $\mu^{+} \tau^{+}$. The analysis requires that two well identified leptons are present in the event: an electron (or muon), and a hadronically decaying tau. For increased sensitivity, the search is split into two exclusive signal detection modes by requiring one or two additional loosely identified lepton candidates. The expected backgrounds for the $e \tau(\mu \tau)$ channels are $0.3(0.4)$ events. No events were observed in the data samples. The limits on $H_{R}^{++}$and $H_{L}^{++}$production and subsequent decay to $e^{+} \tau^{+}$ or $\mu^{+} \tau^{+}$are shown in Figure 5. Assuming $100 \%$ decay to the respective final states, the results from the $e \tau(\mu \tau)$ search exclude $m_{H_{L}++}<114 \mathrm{GeV}(112 \mathrm{GeV})$. 

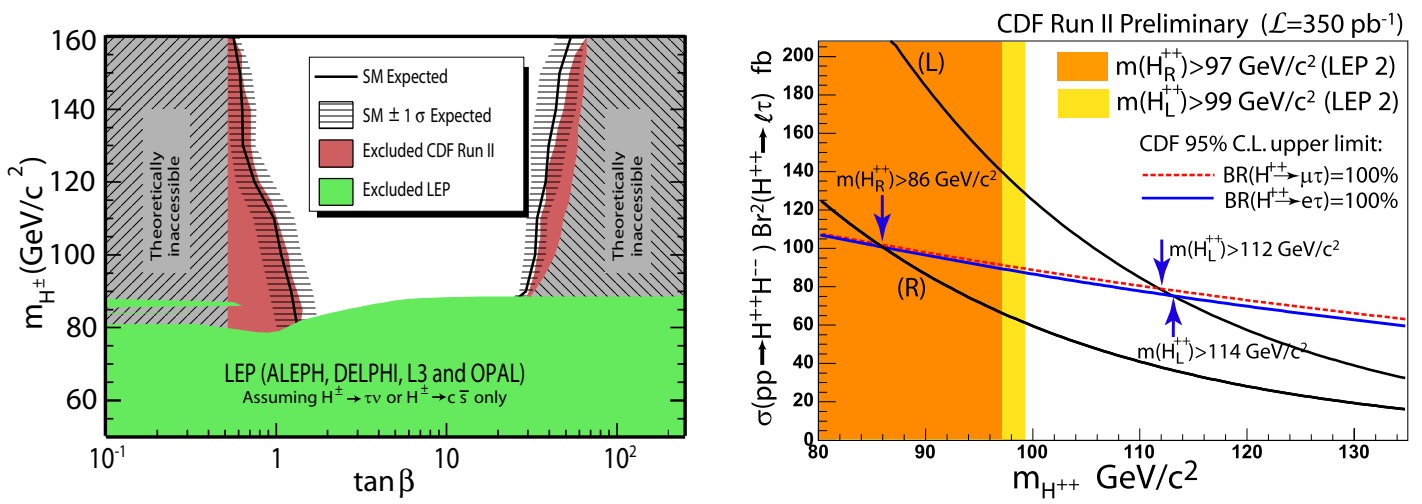

FIGURE 5. Results of the searches for charged Higgs bosons at CDF. Excluded region in the $m_{H^{ \pm}}$vs $\tan \beta$ plane from the search for MSSM $H^{ \pm}$(left), and limits on the doubly-charged Higgs production in Left-Right symmetric models (right).

\section{CONCLUSIONS}

The CDF and DØ collaborations have performed searches for Higgs bosons within the framework of the SM and extended models using data samples corresponding to integrated luminosity of $200-950 \mathrm{pb}^{-1}$. No signal evidence was found and the results were used to set upper limits on Higgs boson production and decay to the investigated final states. The results of searches in the framework of the MSSM are also interpreted as exclusions in the model parameter space.

$\mathrm{CDF}$ and $\mathrm{D} \varnothing$ are working toward improving the analysis techniques and investigating additional signal detection modes to increase the sensitivity of the searches.

\section{REFERENCES}

1. ALEPH, DELPHI, L3, and OPAL Collaborations, Phys.Lett. B565, 61-75 (2003).

2. LEP Electroweak Group, http://lepewwg.web.cern.ch/LEPEWWG/ (2006).

3. D. Acosta et al. (CDF Collaboration), Phys. Rev. D71, 032001 (2005).

4. DØ Collaboration, Nucl. Instrum. Meth., A565, 463-537 (2006).

5. T. Han and S. Willenbrock, Phys. Lett. B273, 167 (1991); M. Spira, hep-ph/9510347 (1995).

6. A. Djouadi, J. Kalinowski, and M. Spira, Comput.Phys.Commun. 108, 56-74 (1998).

7. The plots are courtesy of John Conway.

8. V. Abasov et al. (DØ Collaboration), Phys. Rev. Lett. 97, 161803 (2006).

9. A. Abulencia et al., (CDF Collaboration), Phys. Rev. Lett. 97, 081802 (2006).

10. V. Abasov et al. (DØ Collaboration), Phys. Rev. Lett. 95, 151801 (2005).

11. A. Abulencia et al., (CDF Collaboration), Phys.Rev.Lett. 96, 011802 (2006).

12. V. Abasov et al. (DØ Collaboration), Phys.Rev.Lett. 97, 121802 (2006).

13. M. Carena, S. Heinemeyer, C.E.M. Wagner, G. Weiglein, Eur.Phys.J. C45, 797-814 (2006).

14. M. Carena, S. Heinemeyer, C.E.M. Wagner, G. Weiglein, hep-ph/9912223 (1999); Eur.Phys.J. C26, 601 (2003).

15. A. Abulencia et al., (CDF Collaboration), Phys. Rev. Lett. 96, 042003 (2006). 